\title{
Non-Adiabatic Phonon Dispersion of Metallic Single-Walled Carbon Nanotubes
}

\author{
Valentin N. Popov ${ }^{1}(\bowtie)$, and Philippe Lambin ${ }^{2}$ \\ ${ }^{1}$ Faculty of Physics, University of Sofia, BG-1164 Sofia, Bulgaria \\ ${ }^{2}$ Research Center in Physics of Matter and Radiation, Facultés Universitaires Notre Dame de la Paix, B-5000 Namur, Belgium \\ Received: 6 August 2010 / Revised: 23 September 2010 / Accepted: 23 September 2010 \\ (C) The Author(s) 2010. This article is published with open access at Springerlink.com
}

\begin{abstract}
Non-adiabatic effects can considerably modify the phonon dispersion of low-dimensional metallic systems. Here, these effects are studied for the case of metallic single-walled carbon nanotubes using a perturbative approach within a density-functional-based non-orthogonal tight-binding model. The adiabatic phonon dispersion was found to have logarithmic Kohn anomalies at the Brillouin zone center and at two mirror points inside the zone. The obtained dynamic corrections to the adiabatic phonon dispersion essentially modify and shift the Kohn anomalies as exemplified in the case of nanotube $(8,5)$. Large corrections have the longitudinal optical phonon, which gives rise to the so-called $\mathrm{G}^{-}$band in the Raman spectra, and the carbon hexagon breathing phonon. The results obtained for the $\mathrm{G}^{-}$band for all nanotubes in the diameter range from 0.8 to $3.0 \mathrm{~nm}$ can be used for assignment of the high-frequency features in the Raman spectra of nanotube samples.
\end{abstract}

\section{KEYWORDS}

Nanotube, phonons, G band, tight-binding model

\section{Introduction}

Recently, significant progress has been achieved in the separation of single-walled carbon nanotubes [1-3] and the application of nanotubes with well-defined structure in nanoelectronic devices [4]. Single-walled carbon nanotubes (or, simply, nanotubes) have a honeycomb cylindrical structure consisting entirely of carbon atoms, which determines their unique physical properties. Characterization of the nanotubes usually relies on low-frequency Raman spectra of the radialbreathing band combined with theoretical predictions [5]. For a long time, the intense high-frequency Raman $G$ band has not been used for such purposes except for identification of the nanotubes as metallic or semiconducting [6]. The recent theoretical predictions of the $G$ band frequency in the adiabatic approximation [7] and with dynamic corrections [8, 9], have made it possible to use the experimental data from this band to support the nanotube characterization [10]. The behavior of the $G$ band with varying temperature [11], strain [12], and charge doping [13] provides additional important information about the nanotubes under different conditions.

In low-defect nanotubes at low temperatures, the electron momentum relaxation time can be larger than the characteristic phonon period. Under such conditions, the adiabatic approximation fails and phonon calculations must be performed together with the electronic ones. The effects of non-adiabaticity

Address correspondence to vpopov@phys.uni-sofia.bg 
can be observed in metallic nanotubes, in which electrons (holes) can be scattered by phonons into empty (occupied) electronic states close to the Fermi energy. These processes effectively renormalize the phonons, a phenomenon known as the Kohn anomaly. In nanotubes, the renormalization is expressed in logarithmic softening and increased linewidth of certain phonon branches at low temperatures [8]. The correct description of this anomaly requires going beyond the adiabatic approximation and explicitly accounting for the dynamic effects. These effects were found to be large for the longitudinal optical phonon, which gives rise to the $\mathrm{G}^{-}$band in the Raman spectra of metallic nanotubes, as well as for the carbon hexagon breathing phonon, which is of major importance for double resonance scattering processes in nanotubes. The theoretical work done so far focuses on the Kohn anomalies of the phonon dispersion close to these phonons using an electron zone-folding scheme within an $a b$ initio approach and a chirality-independent curvature correction for the $\mathrm{G}^{-}$-band frequencies [8]. From a practical point of view, knowledge of the precise diameter- and chirality-dependence of the $\mathrm{G}^{-}$ band can be used to support the assignment of the Raman scattering data. As far as we are aware, there have been no large-scale theoretical investigations of the Kohn anomaly in nanotubes for the optimized nanotube structure.

Here, we calculate the dynamic corrections to the phonon dispersion of the metallic nanotube $(8,5)$ using a density-functional-based symmetry-adapted non-orthogonal tight-binding (NTB) model [7]. We also derive the $\mathrm{G}^{-}$band frequency and linewidth for all metallic nanotubes with diameters in the range $0.8 \mathrm{~nm}$ to $3.0 \mathrm{~nm}$.

\section{Theoretical part}

A nanotube can be viewed as an infinite strip of graphene rolled up into a seamless cylinder [7]. The strip has one-dimensional periodicity with a unit cell of $N$ pairs of carbon atoms and a translation period $T$. The Brillouin zone of the strip is a rectangle in the reciprocal space of graphene. The rolling of the strip into a nanotube reduces the Brillouin zone down to $N$ equidistant lines in the rectangle, parallel to the nanotube axis-so-called cutting lines. The electronic states of a nanotube are indexed by the onedimensional wave vector $k(-0.5 \leqslant k<0.5$ in units of $2 \pi / T)$ running along a given cutting line and the linear azimuthal quantum number $l(-N / 2 \leqslant l<N / 2)$ labeling the cutting lines. In the same way, the phonons of a nanotube are indexed by the one-dimensional wave vector $q(-0.5 \leqslant q<0.5$ in units of $2 \pi / T)$ and the linear azimuthal quantum number $\lambda(\lambda=0,1, \ldots, N-1)$. The cutting line with $l=0(\lambda=0)$ passes through the $\Gamma$ point of graphene.

The band structure and the phonon dispersion of the graphene strip and, therefore, of the nanotube with neglect of its curvature, can be related to those of graphene by the zone-folding method. Graphene is characterized by two Dirac cones (conical valence and conduction bands with a common axis and a common apex at the Fermi energy) located at two non-equivalent $\mathrm{K}$ points of the Brillouin zone. Thus, there are states in the valence and conduction bands, between which an electron (hole) can be scattered by a phonon. These scattering processes are enhanced for phonons of the longitudinal optical (LO) and transverse optical (TO) branches close to the $\Gamma$ point (or $\mathrm{LO}(\Gamma)$ and $\mathrm{TO}(\Gamma)$ branches), and the transverse optical branch close to the $\mathrm{K}$ point (or $\mathrm{TO}(\mathrm{K})$ branch). Accounting for these processes in the adiabatic approximation yields Kohn anomalies as finite slopes of the $\mathrm{LO}(\Gamma)$ and $\mathrm{TO}(\mathrm{K})$ branches at the $\Gamma$ and $\mathrm{K}$ points, respectively $[7,14]$. The phonons of these branches at the two points have symmetry $E_{2 g}$ and $A_{1}^{\prime}$, and are often referred to as the $G$ mode and the $A_{1}^{\prime}$ mode, respectively. The non-adiabatic $\mathrm{LO}(\Gamma)$ and $\mathrm{TO}(\mathrm{K})$ branches are flat around the $\Gamma$ and $K$ points, and the $\mathrm{TO}(\Gamma)$ branch has a dip close to the $\Gamma$ point [15], while the $G$ and $A_{1}^{\prime}$ modes are not affected by the dynamic corrections [15-17].

On average, for one third of the nanotubes there are two cutting lines through the two non-equivalent $\mathrm{K}$ points and, therefore, such nanotubes are zero-gap semiconductors (or metallic, $\mathrm{M}$ ). The remaining twothirds of the nanotubes are semiconducting (S). Similarly to graphene, the presence of states in the valence and conduction bands around the $\mathrm{K}$ points with energy separation of the order of the phonon energy favors scattering of electrons (holes) by phonons between 
such states. These processes take place with conservation of energy, wave vector, and azimuthal quantum number in the extended Brillouin zone [18]. In particular, electrons with wave vectors close to the wave vectors $\pm k^{*}$ of the $\mathrm{K}$ points can be scattered by phonons with wave vectors close to 0 and $\pm q^{*}= \pm 2 k^{*}$. If we denote the azimuthal quantum number of the electrons by $\pm l^{*}$, the azimuthal quantum number of the scattering phonon can be 0 and $\pm \lambda^{*}= \pm 2 l^{*}$. It can be demonstrated geometrically that the cutting lines with $\pm \lambda^{*}$ pass through $\mathrm{K}$ points at wave vectors $\pm q^{*}$. Therefore, we can refer to the phonons with $q=0$ and $\lambda=0$ as $\Gamma$ phonons, and to those with $\pm q^{*}$ and $\pm \lambda^{*}$ as $\mathrm{K}$ phonons. The scattering processes are enhanced for phonons belonging to the $\mathrm{LO}$ and $\mathrm{TO}$ branches for cutting lines through the $\Gamma$ and $\mathrm{K}$ points. Later on, these branches and phonons will be referred to using the notation introduced above for graphene, i.e., $\mathrm{LO}(\Gamma)$, $\mathrm{TO}(\Gamma)$, and $\mathrm{TO}(\mathrm{K})$ branches, and $\mathrm{G}$ and $\mathrm{A}_{1}^{\prime}$ modes. The nanotube curvature removes the degeneracy of the $\mathrm{G}$ band, splitting it into lower- and higher-frequency components usually denoted by $\mathrm{G}^{-}$and $\mathrm{G}^{+}$, respectively. The adiabatic $\mathrm{LO}(\Gamma)$ and $\mathrm{TO}(\mathrm{K})$ branches have Kohn anomalies in the form of logarithmic singularities at the $\Gamma$ and $\mathrm{K}$ points $[7,8]$. The non-adiabatic $\mathrm{LO}(\Gamma)$ and $\mathrm{TO}(\mathrm{K})$ branches were found to have zero slopes at the $\Gamma$ and $\mathrm{K}$ points and singularities close to these points [8].

The phonon renormalization can be estimated by calculating the adiabatic phonon dispersion and then determining the dynamic corrections to it in lowestorder perturbation theory. The adiabatic phonon dispersion of a nanotube can be derived from the expansion of the deformation energy of the nanotube due to a static perturbation (a "frozen" phonon) up to second order in the atomic displacements [7]. This expansion contains the adiabatic electronic response to the atomic motion through the phonon self-energy with neglected frequency dependence. The phonon self-energy is given by the sum over the Brillouin zone of the ratio of the squared electron-phonon interaction matrix elements in the numerator and the difference of valence $(v)$ and conduction $(c)$ band energies, $E_{k+q c}-E_{k v}$, in the denominator.

The non-adiabatic phonon frequency, $\omega$, and the phonon linewidth due electron-phonon interactions,
$\Delta \Gamma$, can be derived from the adiabatic dynamical matrix in lowest-order perturbation theory using the expression [14, 19]

$$
(\omega+i \Delta \Gamma)^{2}=\mathbf{e}_{0}^{+} D \mathbf{e}_{0}
$$

Here, $D$ is the dynamical matrix with $\pm \hbar \omega_{0} \pm i \delta$ added to the denominator of the phonon self-energy, where $\omega_{0}$ is the adiabatic phonon frequency and $\delta$ is a small positive number; $\mathbf{e}_{0}$ is the adiabatic phonon eigenvector.

\section{Results and discussion}

The adiabatic phonon dispersion of the nanotubes was derived within the NTB model [7]. The calculations were performed for the optimized nanotube structure and, therefore, the results include both confinement and curvature effects. The obtained phonon dispersion corresponds well to the available experimental data except for overestimation of the high-frequency phonon branches by $11 \%$, but the agreement with experiment is improved if the dispersion is downscaled by a factor of 0.9. For this reason, all presented frequencies are downscaled by this factor. The temperature dependence of the dispersion was introduced by smearing the electron occupation by use of the Fermi-Dirac distribution function for temperature of $300 \mathrm{~K}$.

The calculated adiabatic phonon dispersion of the narrow metallic nanotube $(8,5)$ with diameter of about $0.9 \mathrm{~nm}$ (Fig. 1) has Kohn anomalies in the form of smeared logarithmic softening of the $\mathrm{LO}(\Gamma)$ and $\mathrm{TO}(\mathrm{K})$ branches at the Brillouin zone center and at wave vector $q^{*} \approx 0.21$. It is easy to prove that the zerotemperature anomalies are logarithmic by direct integration over $k$ in Eq. (A1) (see the Appendix) with removed frequency dependence. Previously, dynamic corrections have been found to modify significantly the $\mathrm{LO}(\Gamma), \mathrm{TO}(\Gamma)$, and $\mathrm{TO}(\mathrm{K})$ branches [8]. The modified Kohn anomalies of these branches can be described approximately at zero temperature by the anomalous contribution to the dynamical matrix (see the Appendix)

$$
\bar{D}_{1} \sim-\frac{\left(v_{\mathrm{F}} q\right)^{2}}{\left(v_{\mathrm{F}} q\right)^{2}-\left(\hbar \omega_{0}\right)^{2}}
$$

for the $\mathrm{TO}(\Gamma)$ branch and 


$$
\bar{D}_{1} \sim \ln \left|\left(v_{\mathrm{F}} q-\hbar \omega_{0}\right)\left(v_{\mathrm{F}} q+\hbar \omega_{0}\right)\right|
$$

for the $\mathrm{LO}(\Gamma)$ and $\mathrm{TO}(\mathrm{K})$ branches with $q=0$ and $q^{*}$, respectively. It is clear from Eqs. (2) and (3) that the phonon frequencies have anomalous behavior around $\pm q^{* *}$ and the four points $\pm q^{*} \pm q^{* *}$, where $q^{* *}=\hbar \omega_{0} / v_{\mathrm{F}}$. For nanotube $(8,5), q^{* *} \approx 0.11$ at the $\Gamma$ point and $q^{* *} \approx$ 0.08 at the K point.

The Kohn anomalies, predicted by the simple expressions above, can be observed in the calculated non-adiabatic branches. The dynamic corrections to the $\mathrm{TO}(\Gamma)$ branch are small for the $\mathrm{G}^{+}$mode but are responsible for the singularity at $q^{* *}$ (Fig. 2). The sawlike structure at this wave vector originates from the slightly different Fermi velocities and electron-phonon interaction matrix elements for the two bands, crossing at the Fermi energy. The dynamic corrections to the $\mathrm{LO}(\Gamma)$ and $\mathrm{TO}(\mathrm{K})$ branches are large for the $\mathrm{G}^{-}$mode (Fig. 2) and $\mathrm{A}_{1}^{\prime}$ mode (Fig. 3) but also yield singularities at $q^{* *}$. The anomalies in the three branches disappear away from $q^{* *}$, where the non-adiabatic branches coincide with the adiabatic ones.

The dynamic corrections to the linewidth of the $\mathrm{LO}(\Gamma), \mathrm{TO}(\Gamma)$, and $\mathrm{TO}(\mathrm{K})$ branches can easily be derived

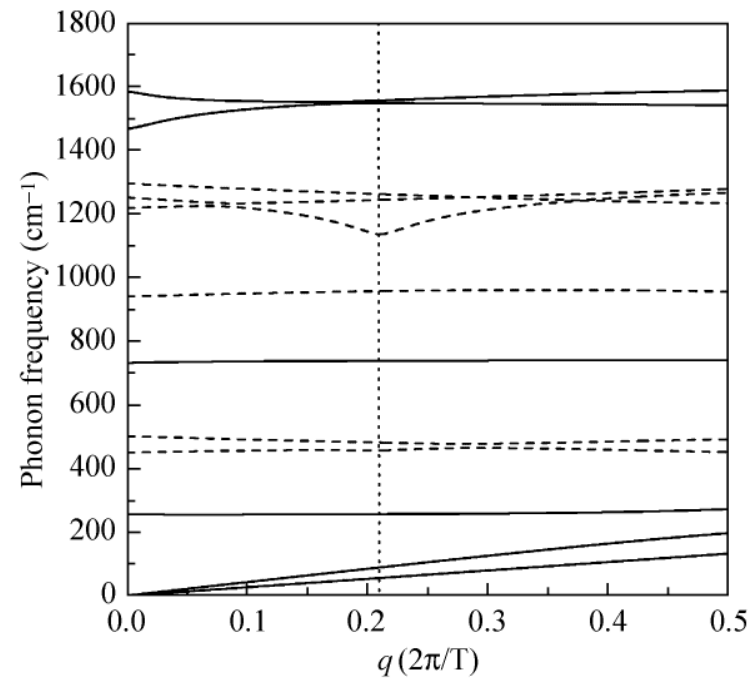

Figure 1 Phonon dispersion of nanotube $(8,5)$. The phonon branches for cutting lines through the $\Gamma$ and $\mathrm{K}$ points of the Brillouin zone of graphene are drawn by solid and dashed lines, respectively. The branches for all other cutting lines are omitted. The phonon dispersion has Kohn anomalies at the $\Gamma$ point $(q=0)$ and $\mathrm{K}$ point $\left(q=q^{*}\right)$. The position of the $\mathrm{K}$ point is marked with a dotted line at zero temperature (see the Appendix)

$$
\Delta \Gamma \sim \delta\left[\left(v_{\mathrm{F}} q-\hbar \omega_{0}\right)\left(v_{\mathrm{F}} q+\hbar \omega_{0}\right)\right]
$$

for the $\mathrm{TO}(\Gamma)$ branch and

$$
\Delta \Gamma \sim H\left[\left(-v_{\mathrm{F}} q+\hbar \omega_{0}\right)\left(v_{\mathrm{F}} q+\hbar \omega_{0}\right)\right]
$$

for the $\mathrm{LO}(\Gamma)$ and $\mathrm{TO}(\mathrm{K})$ branches with $q=0$ and $q^{*}$, respectively. Thus, the linewidth for the $\mathrm{TO}(\Gamma)$ branch is a Dirac $\delta$-function, centered at $\pm q^{* *}$ and those for the $\mathrm{LO}(\Gamma)$ and $\mathrm{TO}(\mathrm{K})$ branches are rectangular functions of width $2 q^{* *}$, centered at $q=0$ and $\pm q^{*}$, respectively. Finite temperature smears the linewidth of both functions (Figs. 2 and 3). The linewidth for the $\mathrm{TO}(\Gamma)$ branch has the shape of a broadened Dirac $\delta$-function (Fig. 2) with two spikes, which are due to the differences between the two bands, crossing at the Fermi
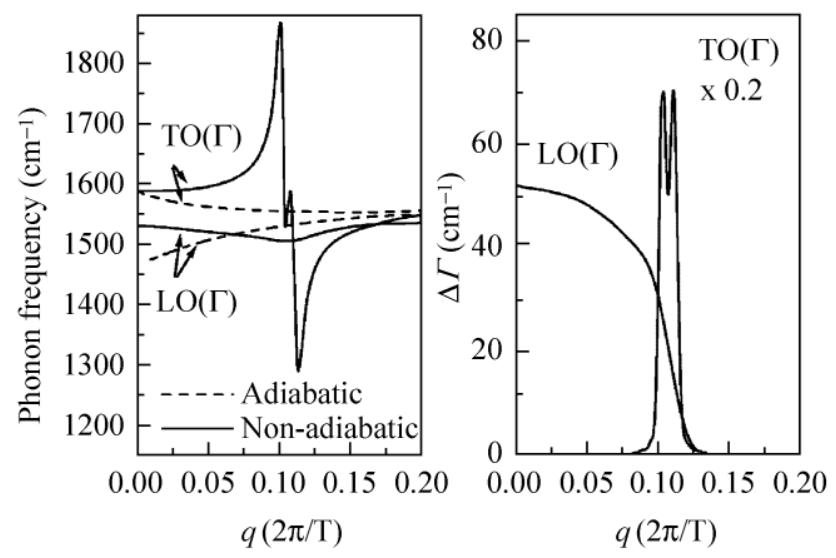

Figure $2 \mathrm{LO}(\Gamma)$ and $\mathrm{TO}(\Gamma)$ branch frequency (left) and linewidth $\Delta \Gamma$ (right) of nanotube $(8,5)$ in the vicinity of the $\Gamma$ point $(q=0)$
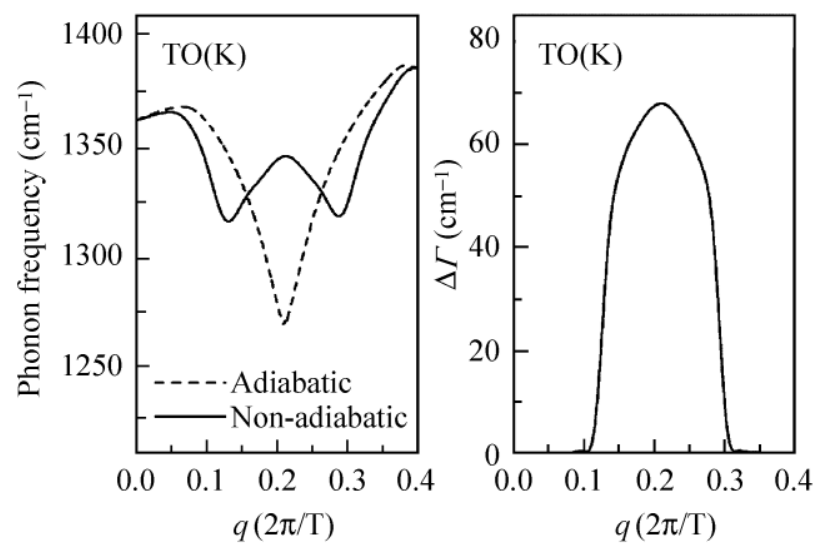

Figure $3 \mathrm{TO}(\mathrm{K})$ branch frequency (left) and linewidth $\Delta \Gamma$ (right) of nanotube $(8,5)$ in the vicinity of the $\mathrm{K}$ point $\left(q=q^{*}\right)$ 
energy, as mentioned above. The linewidths for the $\mathrm{LO}(\Gamma)$ and $\mathrm{TO}(\mathrm{K})$ branches are smeared rectangular functions (Figs. 2 and 3). The similarity of the behavior of the dynamic corrections to the latter two branches can be explained by allowed backscattering of electrons, while the behavior of the $\mathrm{TO}(\Gamma)$ branch stems from allowed forward scattering of electrons. The ratio of the linewidths of the $G^{-}$and $A_{1}^{\prime}$ modes, derived from Eq. (A7) (in the Appendix), is

$$
\frac{2 \omega_{0}\left(\mathrm{~A}_{1}^{\prime}\right)\left(M_{\mathrm{LO}}^{\Gamma}\right)^{2}}{\omega_{0}\left(\mathrm{G}^{-}\right)\left(M_{\mathrm{TO}}^{\mathrm{K}}\right)^{2}} \approx 1.25
$$

where $M_{\mathrm{LO}}^{\Gamma}=12.8 \mathrm{eV} \cdot \AA^{-1}$ and $M_{\mathrm{TO}}^{\mathrm{K}}=18.0 \mathrm{eV} \cdot \AA^{-1}[18]$. This ratio is in good agreement with the calculated one of 1.28 .

The obtained $\mathrm{G}^{-}$and $\mathrm{G}^{+}$mode frequencies for all metallic nanotubes with diameters $d$ between $0.8 \mathrm{~nm}$ and $3.0 \mathrm{~nm}$ are shown in Fig. 4 in comparison with the data for all semiconducting nanotubes in the same range [7]. The frequencies can be grouped in two strips, which correspond to the observed Raman bands of nanotube samples: the $\mathrm{G}^{+}$band at about $1590 \mathrm{~cm}^{-1}$ and the $\mathrm{G}^{-}$band in the range $1540-1570 \mathrm{~cm}^{-1}([6])$.

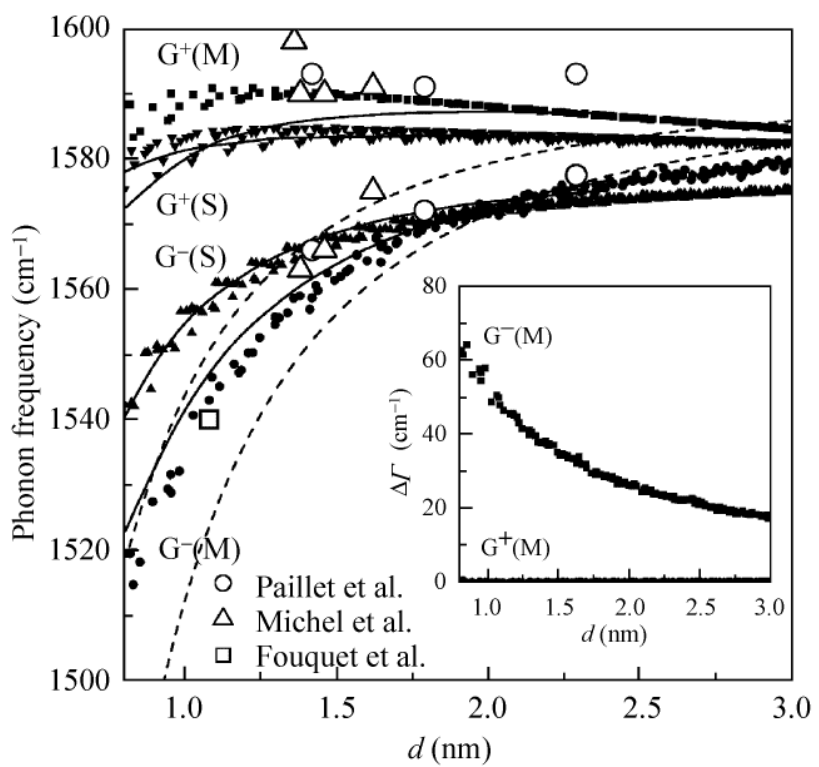

Figure $4 \mathrm{G}^{-}$and $\mathrm{G}^{+}$mode frequencies for all metallic (M) and semiconducting (S) nanotubes with diameters $d$ from $0.8 \mathrm{~nm}$ to $3.0 \mathrm{~nm}$. The solid lines are DFT predictions [8]. The dashed lines are fits to Raman data [6]. The open symbols are Raman data for individual nanotubes. The inset shows the linewidth of the $\mathrm{G}^{-}$and $\mathrm{G}^{+}$modes for $\mathrm{M}$ nanotubes
Initially, it was generally accepted that the $\mathrm{G}^{-}$band is due to TO modes, while the $\mathrm{G}^{+}$band was ascribed to LO modes, because the softening of the former could come from the mixed $\mathrm{sp}^{2}-\mathrm{sp}^{3}$ hybridization around the nanotube circumference. Theoretical analysis of the separate effects of electron-phonon coupling, confinement, curvature, and dynamic effects, lead to a different conclusion, however [8]. In the simple zonefolding scheme, which describes the effects of confinement, the account of the electron-phonon coupling in the adiabatic approximation yields the LO-TO splitting of the G band. The TO modes remain diameter-independent but the LO modes for M and S nanotubes shift downwards and upwards, respectively $[7,8]$. In $M$ nanotubes, the curvature and dynamic effects result in almost canceling contributions for the TO mode but bend up the LO mode. In $\mathrm{S}$ nanotubes, the curvature effects bend down the LO modes and, more significantly, the TO modes. The behavior of the modes with all effects included is confirmed by our results. Namely, the $\mathrm{G}^{+}$band originates from $\mathrm{G}^{+}(\mathrm{M})$ and $\mathrm{G}^{+}(\mathrm{S})$ modes with transverse and longitudinal polarization, respectively, while the $\mathrm{G}^{-}$band is due to $\mathrm{G}^{-}(\mathrm{M})$ and $\mathrm{G}^{-}(\mathrm{S})$ modes with longitudinal and transverse polarization, respectively. For diameters of about $2 \mathrm{~nm}$, a crossing of the two $\mathrm{G}^{-}$strips is observed, which is in agreement with available ab initio results (see Fig. 19 in Ref. [8]). For smaller diameters, the four strips have the following order of increasing frequency: $\mathrm{G}^{-}(\mathrm{M})<\mathrm{G}^{-}(\mathrm{S})<\mathrm{G}^{+}(\mathrm{S})<\mathrm{G}^{+}(\mathrm{M})$, in agreement with previous predictions $[8,9]$. The dependence of the $\mathrm{G}^{-}$ frequency (in $\mathrm{cm}^{-1}$ ) on the diameter $d$ (in $\mathrm{nm}$ ) can be fitted by the chirality-independent expression [6]

$$
\omega=\omega_{\infty}-\frac{A}{d^{2}}
$$

Here, $\omega_{\infty}=1582 \mathrm{~cm}^{-1}$ and $A=44.76 \mathrm{~nm}^{2} \cdot \mathrm{cm}^{-1}$ for $\mathrm{M}$ nanotubes, and $\omega_{\infty}=1579 \mathrm{~cm}^{-1}$ and $A=23.44 \mathrm{~nm}^{2} \cdot \mathrm{cm}^{-1}$ for $S$ nanotubes. The $\mathrm{G}^{+}(\mathrm{M})$ and $\mathrm{G}^{+}(\mathrm{S})$ mode frequencies are almost diameter-independent with values of about $1591 \mathrm{~cm}^{-1}$ and $1584 \mathrm{~cm}^{-1}$, respectively. Comparison of our results with recent discrete Fourier transform (DFT) data [8] shows excellent agreement except for the $\mathrm{G}^{+}(\mathrm{M})$, where we do not observe significant diameterdependence. The disagreement can be attributed to 
the use of an approximate scheme for correction of the zone-folding DFT data. The fit to extensive experimental data on isolated nanotubes on a substrate yielded $\omega_{\infty}=1591 \mathrm{~cm}^{-1}$ and $A=47.7 \mathrm{~nm}^{2} \cdot \mathrm{cm}^{-1}$ for S nanotubes, and $A=79.5 \mathrm{~nm}^{2} \cdot \mathrm{cm}^{-1}$ for $\mathrm{M}$ nanotubes [6]. The latter fits give lower values for narrow nanotubes and higher values for wide nanotubes compared to the NTB and DFT predictions. The experimental data for the $\mathrm{G}^{+}$ bands in Ref. [6] were fitted by a horizontal line at $1591 \mathrm{~cm}^{-1}$, while the NTB and DFT results show softening of these bands for narrow nanotubes.

Raman data for isolated, well-identified nanotubes, are still quite rare. Combined electron diffraction and Raman scattering methods allowed the identification of freestanding $S$ nanotubes on a metallic grid with indices, diameters, $\mathrm{G}^{-}$and $\mathrm{G}^{+}$bands, as follows: $(12,8)$, $1.38 \mathrm{~nm}, 1563 \mathrm{~cm}^{-1}, 1590 \mathrm{~cm}^{-1}$ [10]; (11,10), $1.42 \mathrm{~nm}$, $1566 \mathrm{~cm}^{-1}, 1593 \mathrm{~cm}^{-1},(17,9), 1.79 \mathrm{~nm}, 1572 \mathrm{~cm}^{-1}$, $1591 \mathrm{~cm}^{-1}$, and $(27,4), 2.29 \mathrm{~nm}, 1577.5 \mathrm{~cm}^{-1}, 1593 \mathrm{~cm}^{-1}$ [20]. Recently, similar measurements have been performed on M nanotubes: (10,10), $1.36 \mathrm{~nm}$, (the $\mathrm{G}^{-}$mode is not Raman active), $1598 \mathrm{~cm}^{-1},(15,6), 1.46 \mathrm{~nm}$, $1566 \mathrm{~cm}^{-1}, 1590 \mathrm{~cm}^{-1}$, and $(19,16), 1.62 \mathrm{~nm}, 1575 \mathrm{~cm}^{-1}$, $1591 \mathrm{~cm}^{-1}$ [10]. In Raman and Rayleigh scattering experiments on nanotubes grown across a wide slit [21] the $G^{-}$band of the metallic nanotube $(12,3)$ with $d=1.08 \mathrm{~nm}$ was observed at $1540 \mathrm{~cm}^{-1}$. Our calculations correspond well to these experimental data for the $\mathrm{G}^{-}(\mathrm{M}), \mathrm{G}^{-}(\mathrm{S})$, and $\mathrm{G}^{+}(\mathrm{M})$ bands, while both NTB and DFT results systematically underestimate the $\mathrm{G}^{+}(\mathrm{S})$ band by several $\mathrm{cm}^{-1}$. We note that this deviation cannot be explained by temperature and strain, which would shift the experimental values downward. In order to resolve this disagreement, more theoretical work has to be done, and also more experimental data on well-identified nanotubes need to be collected.

The inset of Fig. 4 shows that the linewidth of the $\mathrm{G}^{-}(\mathrm{M})$ modes decreases monotonically with increasing diameter following roughly a $c / d$ law with $c=$ $53 \mathrm{~nm} \cdot \mathrm{cm}^{-1}$. The analytical formula, Eq. (A7) (see the Appendix), with the large-diameter values $\omega_{0}=1590$ $\mathrm{cm}^{-1}$ and $M=12.8 \mathrm{eV} \cdot \AA^{-1}$ (reduced by a factor of 0.9 [15]) yields $c=59 \mathrm{~nm} \cdot \mathrm{cm}^{-1}$. The deviation of the latter from the former may be due to the approximations in the analytical formula and temperature effects on the computed linewidth. In the small-diameter region, $\Delta \Gamma$ has chirality dependence. The use of DFT data [22] in the same equation yields larger value $c=79 \mathrm{~nm} \cdot \mathrm{cm}^{-1}$ because of the larger value of the matrix element $M=$ $13.5 \mathrm{eV} \cdot \AA^{-1}$. The measured linewidths show a wide spread, and verification of the true value of $c$ is not unambiguous.

\section{Conclusions}

We have performed NTB calculations of the phonon dispersion of the metallic nanotube $(8,5)$ and, in particular, studied the non-adiabatic modification of the Kohn anomalies in the vicinity of the $\Gamma$ and $\mathrm{K}$ points of the Brillouin zone. We reproduced the $a b$ initio results for the LO and TO phonons close to the $\Gamma$ point and for the TO phonons close to the $\mathrm{K}$ point. We derived $\mathrm{G}^{-}$and $\mathrm{G}^{+}$mode frequencies for all metallic nanotubes with diameters between 0.8 and $3.0 \mathrm{~nm}$. The results for the $G$ bands can be used for assignment of the high-frequency lines of the Raman spectra of nanotube samples.

\section{Acknowledgements}

V. N. P. was supported partly by the Marie Curie European Reintegration Grant No. MERG-CT-2007201227 within the $7^{\text {th }}$ European Community Framework Programme and partly by NSF under grant No. DO 02-136/15.12.2008 (IRC-CoSiM).

Open Access: This article is distributed under the terms of the Creative Commons Attribution Noncommercial License which permits any noncommercial use, distribution, and reproduction in any medium, provided the original author(s) and source are credited.

\section{References}

[1] Tanaka, T.; Jin, H.; Miyata, Y.; Fujii, S.; Suga, H.; Naitoh, Y.; Minari, T.; Miyadera, T.; Tsukagoshi, K.; Kataura, H. Simple and scalable gel-based separation of metallic and semiconducting carbon nanotubes. Nano Lett. 2009, 9, 14971500 .

[2] Tu, X.; Manohar, S.; Jagota, A.; Zheng, M. DNA sequence motifs for structure-specific recognition and separation of carbon nanotubes. Nature 2009, 460, 250-253.

\section{(10) Springer}


[3] Stürzl, N.; Hennrich, F.; Lebedkin, S.; Kappes, M. M. Near monochiral single-walled carbon nanotube dispersions in organic solvents. J. Phys. Chem. C 2009, 113, 14628-14632.

[4] Vijayaraghavan, A.; Hennrich, F.; Stürzl, N.; Engel, M.; Ganzhorn, M.; Oron-Carl, M.; Marquardt, C. W.; Dehm, S.; Lebedkin, S.; Kappes, M. M.; Krupke, R. Toward singlechirality carbon nanotube device arrays. ACS Nano 2010, 4, 2748-2754.

[5] Dresselhaus, M. S.; Jorio, A.; Hofmann, M.; Dresselhaus, G.; Saito, R. Perspectives on carbon nanotubes and graphene Raman spectroscopy. Nano Lett. 2010, 10, 751-758.

[6] Jorio, A.; Souza Filho, A. G.; Dresselhaus, G.; Dresselhaus, M. S.; Swan, A. K.; Ünlü, M. S.; Goldberg, B. B.; Pimenta, M. A.; Hafner, J. H.; Lieber, C. M.; Saito, R. G-band resonant Raman study of 62 isolated single-wall carbon nanotubes. Phys. Rev. B 2002, 65, 155412.

[7] Popov, V. N.; Lambin, Ph. Radius and chirality dependence of the radial breathing mode and the G-band phonon modes of single-walled carbon nanotubes. Phys. Rev. B 2006, 73, 085407.

[8] Piscanec, S.; Lazzeri, M.; Robertson, J.; Ferrari, A. C.; Mauri, F. Optical phonons in carbon nanotubes: Kohn anomalies, Peierls distortions, and dynamic effects. Phys. Rev. B 2007, 75, 035427.

[9] Sasaki, K.; Saito, R.; Dresselhaus, G.; Dresselhaus, M. S.; Farhat, H.; Kong, J. Curvature-induced optical phonon frequency shift in metallic carbon nanotubes. Phys. Rev. B 2008, 77, 245441.

[10] Michel, T.; Paillet, M.; Nakabayashi, D.; Picher, M.; Jourdain, V.; Meyer, J. C.; Zahab, A. A.; Sauvajol, J. L. Indexing of individual single-walled carbon nanotubes from Raman spectroscopy. Phys. Rev. B 2009, 80, 245416.

[11] Zhang, L.; Jia, Z.; Huang, L.; O'Brien, S.; Yu, Z. Lowtemperature Raman spectroscopy of individual single-wall carbon nanotubes and single-layer graphene. J. Phys. Chem. C 2008, 112, 13893-13900.

[12] Wang, B.; Gupta, A. K.; Huang, J.; Vedala, H.; Hao, Q.; Crespi, V. H.; Choi, W.; Eklund, P. C. Effect of bending on single-walled carbon nanotubes: A Raman scattering study. Phys. Rev. B 2010, 81, 115422.

[13] Kalbac, M.; Farhat, H.; Kavan, L.; Kong, J.; Sasaki, K.; Saito, R.; Dresselhaus, M. S. Electrochemical charging of individual single-walled carbon nanotubes. ACS Nano 2009, 3, 2320-2328.

[14] Piscanec, S.; Lazzeri, M.; Mauri, F.; Ferrari, A. C.; Robertson, J. Kohn anomalies and electron-phonon interactions in graphite. Phys. Rev. Lett. 2004, 93, 185503.

[15] Popov, V. N.; Lambin, Ph. Dynamic and charge doping effects on the phonon dispersion of graphene. Phys. Rev. B 2010, 82,045406 .
[16] Pisana, S; Lazzeri, M.; Casiraghi, C.; Novoselov, K. S.; Geim, A. K.; Ferrari, A. C.; Mauri, F. Breakdown of the adiabatic Born-Oppenheimer approximation in graphene. Nat. Mater. 2007, 6, 198-201.

[17] Yan, J.; Zhang, Y.; Kim, Ph.; Pinczuk, A. Electric field effect tuning of electron-phonon coupling in graphene. Phys. Rev. Lett. 2007, 98, 166802

[18] Popov, V. N.; Lambin, Ph. Intraband electron-phonon scattering in single-walled carbon nanotubes. Phys. Rev. B 2006, 74, 075415.

[19] Haken, H. Quantum Field Theory of Solids: An Introduction; North-Holland: Amsterdam, 1976.

[20] Paillet, M.; Michel, T.; Meyer, J. C.; Popov, V. N.; Henrard, L.; Roth, S.; Sauvajol, J. L. Raman active phonons of identified semiconducting single-walled carbon nanotubes. Phys. Rev. Lett. 2006, 96, 257401.

[21] Fouquet, M.; Telg, H.; Maultzsch, J.; Wu, Y.; Chandra, B.; Hone, J.; Heinz, T. F.; Thomsen, C. Longitudinal optical phonons in metallic and semiconducting carbon nanotubes. Phys. Rev. Lett. 2009, 102, 075501.

[22] Lazzeri, M.; Piscanec, S.; Mauri, F.; Ferrari, A. C.; Robertson, J. Phonon linewidths and electron-phonon coupling in graphite and nanotubes. Phys. Rev. B 2006, 73, 155425.

\section{Appendix}

The dynamical matrix $D$ of a nanotube consists of terms with second-order variations of the matrix elements of the Hamiltonian and the overlap matrix elements of the NTB model and terms with first-order variations of these matrix elements [7]. The latter contain the response of the electrons to the atomic motion and give rise to Kohn anomalies. The corresponding terms in $\mathbf{e}_{0}^{+} D \mathbf{e}_{0}$ can be written for zero temperature, up to the factor $\alpha T / 2 \pi N m,(\alpha=2$ for $\mathrm{LO}(\Gamma)$ and $\mathrm{TO}(\Gamma)$ phonons, and $\alpha=1$ for $\mathrm{TO}(\mathrm{K})$ phonons; $m$ is the carbon atom mass), as [8]

$$
\bar{D} \equiv \sum_{ \pm} \int_{-\infty}^{+\infty} \frac{M^{2}}{E_{k v}-E_{k+q c} \pm \hbar \omega_{0} \pm i \delta} \mathrm{d} k
$$

where the sum consists of two terms with signs " + " and " - " in the denominator. The scalar quantity $M$ is the product of the electron-phonon matrix element and the phonon eigenvector $\mathbf{e}_{0}$, and generally depends on the conduction and valence bands, as well as on the electron and phonon wave vectors. $\bar{D}$ has singular 
behavior for a vanishing denominator, i.e., for $E_{k+q c}-$ $E_{k v} \sim \hbar \omega_{0}$, in which case it will determine the behavior of the dynamical matrix in the vicinity of the Kohn anomalies. Close to the Fermi energy, $M$ is almost independent of the wave vector and can be assumed to be a constant, which is different for forward scattering (FS) and backscattering (BS) processes. For $\mathrm{LO}(\Gamma)$ phonons, $M=M_{\mathrm{LO}}^{\Gamma}$ for BS and is zero for FS processes; for $\mathrm{TO}(\Gamma)$ phonons, $M=M_{\mathrm{TO}}^{\Gamma}$ for FS and is zero for BS processes; for $\mathrm{TO}(\mathrm{K})$ phonons, $M=M_{\mathrm{TO}}^{\mathrm{K}}$ for $\mathrm{BS}$ and is zero for FS processes. Under these assumptions, the integration in Eq. (A1) can be performed explicitly.

In the vicinity of the Fermi energy, the conduction and valence bands are linear and can be approximated as $E_{k c}= \pm v_{\mathrm{F}} k$ and $E_{k v}= \pm v_{\mathrm{F}} k$ relative to the Fermi energy, where $k$ is the electron wave vector relative to the $\mathrm{K}$ point and $v_{\mathrm{F}}$ is the Fermi velocity, which is assumed to have the same absolute value for both conduction and valence bands. Then, Eq. (A1) can be split into the sum of three integrals over the regions $[-\bar{k},-q],[-q, 0]$, and $[0, \bar{k}]$, where $\bar{k}$ is a large cutoff wave vector, $|\bar{k}| \gg\left|v_{\mathrm{F}} q \pm \hbar \omega_{0}\right|$.

The behavior of the non-adiabatic phonon frequency $\omega$ is determined by the real part $\bar{D}_{1}$ of Eq. (A1). For scattering from $\mathrm{LO}(\Gamma)$ phonons, we get

$$
\begin{aligned}
\bar{D}_{1}= & \sum_{ \pm}\left[\int_{-\bar{k}}^{-q} \frac{\left(M_{\mathrm{LO}}^{\Gamma}\right)^{2}}{v_{\mathrm{F}} k+v_{\mathrm{F}}(k+q) \pm \hbar \omega_{0}} \mathrm{~d} k\right. \\
& \left.+\int_{0}^{+\bar{k}} \frac{\left(M_{\mathrm{LO}}^{\Gamma}\right)^{2}}{-v_{\mathrm{F}} k-v_{\mathrm{F}}(k+q) \pm \hbar \omega_{0}} \mathrm{~d} k\right]
\end{aligned}
$$

It can be easily shown that integration yields the following expression

$$
\bar{D}_{1}=\frac{\left(M_{\mathrm{LO}}^{\Gamma}\right)^{2}}{v_{\mathrm{F}}} \ln \left|\frac{\left(v_{\mathrm{F}} q-\hbar \omega_{0}\right)\left(v_{\mathrm{F}} q+\hbar \omega_{0}\right)}{\left(2 v_{\mathrm{F}} \bar{k}\right)^{2}}\right|
$$

Similarly, for $\mathrm{TO}(\Gamma)$ phonons, we have

$$
\bar{D}_{1}=\sum_{ \pm} \int_{-q}^{0} \frac{\left(M_{\mathrm{TO}}^{\Gamma}\right)^{2}}{v_{\mathrm{F}} k-v_{\mathrm{F}}(k+q) \pm \hbar \omega_{0}} \mathrm{~d} k
$$

and, therefore,

$$
\bar{D}_{1}=-\frac{\left(M_{\mathrm{TO}}^{\Gamma}\right)^{2}}{v_{\mathrm{F}}} \frac{\left(v_{\mathrm{F}} q\right)^{2}}{\left(v_{\mathrm{F}} q\right)^{2}-\left(\hbar \omega_{0}\right)^{2}}
$$

The non-adiabatic $\mathrm{TO}(\mathrm{K})$ phonon frequency is derived in the same way as for the $\mathrm{LO}(\Gamma)$ phonons and the result is similar.

The non-adiabatic contribution to the phonon linewidth is determined by the imaginary part $\bar{D}_{2}$ of Eq. (A1). For scattering from $\mathrm{LO}(\Gamma)$ phonons, we have

$$
\begin{aligned}
\bar{D}_{2}= & \sum_{ \pm}\left[\int_{-\bar{k}}^{-q} \frac{\mp \delta\left(M_{\mathrm{LO}}^{\Gamma}\right)^{2}}{\left(v_{\mathrm{F}}(2 k+q) \pm \hbar \omega_{0}\right)^{2}+\delta^{2}} \mathrm{~d} k\right. \\
& \left.+\int_{0}^{+\bar{k}} \frac{\mp \delta\left(M_{\mathrm{LO}}^{\Gamma}\right)^{2}}{\left(-v_{\mathrm{F}}(2 k+q) \pm \hbar \omega_{0}\right)^{2}+\delta^{2}} \mathrm{~d} k\right]
\end{aligned}
$$

In the limit $\delta \rightarrow 0, \bar{D}_{2}$ tends to $\pi H\left(-v_{\mathrm{F}} q+\hbar \omega_{0}\right)$, where $H(x)$ is the Heaviside step function. The result for both positive and negative $q$ is $\pi H\left[\left(-v_{\mathrm{F}} q+\hbar \omega_{0}\right)\right.$ $\left.\left(v_{\mathrm{F}} q+\hbar \omega_{0}\right)\right]$. The limiting expression of $\bar{D}_{2}$ for the $\mathrm{TO}(\mathrm{K})$ branch is the same as for the $\mathrm{LO}(\Gamma)$ one but with $q$ relative to the $\mathrm{K}$ point. Including the omitted factor in Eq. (A1), we obtain the full width at half maximum of the $\mathrm{G}^{-}$and $\mathrm{A}_{1}^{\prime}$ modes as [20]

$$
\Delta \Gamma=\frac{\alpha A M^{2}}{2 \pi d v_{\mathrm{F}} m \omega_{0}}
$$

where $N=T \pi d / A$ ( $A$ is the unit cell area of graphene).

In the case of $\mathrm{TO}(\mathrm{K})$ phonons, we obtain a similar result. Finally, for scattering from $\mathrm{TO}(\Gamma)$ phonons, we can write

$$
\bar{D}_{2}=\sum_{ \pm} \int_{-q}^{0} \frac{ \pm \delta\left(M_{\mathrm{TO}}^{\Gamma}\right)^{2}}{\left(v_{F} q \pm \hbar \omega_{0}\right)^{2}+\delta^{2}} \mathrm{~d} k
$$

In the limit $\delta \rightarrow 0, \bar{D}_{2}$ tends, up to a power-law factor, to the Dirac delta function $\delta\left(v_{\mathrm{F}} q-\hbar \omega_{0}\right)$. The result for both positive and negative $q$ is $\delta\left[\left(v_{\mathrm{F}} q-\hbar \omega_{0}\right)\left(v_{\mathrm{F}} q+\hbar \omega_{0}\right)\right]$. 